\title{
Network Analysis in Korean Presidential Speeches by Using Word2Vec
}

\author{
Young-Jai PARK* · Young-Bin KIM · Seon-Young JEONG \\ - Young Jin KIM · Seung-Woo SoN ${ }^{\dagger}$ \\ Department of Applied Physics, Hanyang University, Ansan 15588, Korea
}

(Received 10 January 2017 : revised 4 April 2017 : accepted 7 April 2017)

\begin{abstract}
In this paper, using a total of 6,870 presidential speeches from 1948 to 2015, we show the characteristics in the words and analyze the Korean presidential styles. Firstly, we determine how well the distribution of the usage frequency of each word in the speeches follows Zipf's law. The distribution exhibits three slopes that have different properties. Next, by using the distribution data, we compare the trends of the words in the speeches to the actual historical events in Korean politics. Finally, we construct a network among words by using Word2Vec with an artificial neural network and machine learning, and we compare the network to presidential features and the timely change of politics in Korea.
\end{abstract}

PACS numbers: 89.65.-s, 89.75.Da

Keywords: Presidential speeches, Word2Vec, Network among words, Zipf's law

\section{Word2Vec을 이용한 한국 대통령 연설문 네트워크 분석}

\author{
박영재* · 김영빈 · 정선영 · 김영진 · 손승우 \\ 한양대학교 응용물리학과, 안산 15588 , 대한민국
}

(2017년 1월 10일 받음, 2017년 4월 4일 수정본 받음, 2017년 4월 7일 게재 확정)

\begin{abstract}
본 연구에서는 1948 년부터 2015 년까지 총 6,870 편의 한국 대통령 11 명 연설을 통해 연설문에서 사용된 단어의 특징을 살펴보고, 단어간 네트워크를 통해 각 스타일을 분석해보았다. 첫째로는 연설문 내에서 사 용된 단어들이 알려진 지프 법칙 (Zipf's law)을 따르는지 살펴보았다. 단어 빈도수 분포는 크게 세 가지 구간으로 구분되며, 구간마다 그 특징을 살펴보았다. 다음으로는 단어 빈도수를 통해 단어 쓰임의 변화 추이를 분석함으로써, 실제 역사적 배경과 비교해 보았다. 마지막으로 인공신경망과 기계학습을 이용한 Word $2 \mathrm{Vec}$ 방법으로 단어 간의 네트워크 분석을 통하여, 대통령별 특징과 한국의 시대적 변화 과정을 비교해 보았다.
\end{abstract}

PACS numbers: 89.65.-s, 89.75.Da

Keywords: 대통령 연설문, Word2 Vec, 단어 간의 네트워크, 지프 법칙

\footnotetext{
*E-mail: youngjaip0324@gmail.com

†E-mail: sonswoo@hanyang.ac.kr
} 


\section{I. 서 론}

단어를 분석하는데 있어서 한국어는 영어보다 더 복잡 하다. 영어와 달리, 한국어는 교착어적 특성을 지닌 언어로 하나 이상의 형태소가 결합되어 한 어절을 형성하기 때문 이다. 영어의 형태소 분석에는 하나 이상의 단어가 하나의 형태소에 해당하므로, 형태소를 분리하는 일이 없다. 한국 어의 경우 어절을 구성하는 형태소들을 분리해내고, 각 형 태소의 품사를 결정해야 하므로 형태소 분석의 복잡도가 높아진다 [1]. 언어 관련 분야에서는 영어에서 밝혀진 지프 법칙 (Zipf's law)이 다른 언어권에서도 성립하는지에 대한 연구가 계속되고 있으며, 한국어 역시 예외는 아니다 [2].

대통령은 '정치 · 사회', '산업 · 경제', '외교 · 통상', '국 방', '과학기술정보', '교육', '문화 · 체육 · 관광', '환경'에 걸쳐 나라의 상황에 대해 연설문을 발표한다. 2008년 이래 로 한국은 대통령 기록관을 통해 역대 대통령 연설기록들을 국민들에게 공개해왔다 [3]. 대통령 기록관은 1948년 이승 만 정권부터 현 박근혜 정부까지 11 명에 대해 총 6,870 여 편의 연설문을 보관하고 있다.

최근 텍스트 분석이 활발해짐에 따라, 2013년 Mikolov 그룹은 Word2Vec이라는 단어를 벡터로 표현해주는 알고 리즘을 개발하였다 [4]. 이 알고리즘은 인공신경망 (artificial neural network)을 기반으로 하는 기계학습 (machine learning)이며, 최적화 알고리즘으로 분석 시간을 크게 단 축시켰다 $[5]$.

본 연구는 한국 대통령 연설문을 텍스트 분석하여 지프 법칙 (Zipf's law)을 살펴보고, 시대별 빈도수 변화를 관찰 함으로써 역사적 배경과 비교한다. Word2 Vec을 기반으로 연설문 내 단어 네트워크를 구성하고, 대통령별 특징과 한 국의 시대적 변화 과정을 시각화한다.

\section{II. 데이터}

우리나라는 2008 년 4 월 대통령기록관은 개관하고, 역대 대통령 연설기록들을 공개하였다 [3]. 1945년 광복 이래로 우리나라는 초대 대통령 이승만부터 현 대통령인 박근혜 까지 총 11 명이 대통령직을 맡아왔다. 역사적으로 제 3 대 윤보선 전 대통령과 제 10 대 최규하 전 대통령은 그 기간이 짧아 연설문의 갯수가 3 개, 58 개로 적다. 이에 반해 이승만, 박정희, 전두환 전 대통령의 경우는 다른 대통령들과 달리 각각 1-2대, 5-9대, 11-12대 동안 대통령의 자리에 있었다. 각각의 대통령 기간동안의 연설문에 대한 정보를 Table 1 에 정리하였다. 각 연설문의 크기는 텍스트 파일 기준으로 총 $43 \mathrm{MB}$ 이다. 연설문 내에서는 63,225 종류의 단어들이 총 $8,741,085$ 회 쓰였다.

\section{III. 방 법}

\section{1. 지프 법칙 (Zipf's law)}

1940년대 하버드 대학의 조지 킹슬리 지프 (George Kingsley Zipf) 교수는 미국 신문에서 특정 단어를 얼마 나 자주 사용했는지 세어 보았다. 그 결과 10 위의 단어는 2,653 번 등장했으며, 20 위의 단어는 1,311 번 등장했다. 2 만 번째 단어는 단 한 번 등장했다. 지프 법칙 (Zipf's law) 은 한 데이터 안에서 단어의 빈도수를 순위별로 정리했을 때, 다음과 같은 거듭제곱법칙 (power law)을 따르게 되는 것을 말한다 [6].

$$
Z(r) \sim r^{-\alpha}
$$

특히 지프 법칙에서 각 축의 축척을 로그 단위로 표현 할 경우, 빈도-순위 그래프의 개형이 직선이 되며, 특히 그 기울기가 대략 $-1(\alpha \sim 1)$ 을 따른다.

$$
\log Z(r) \sim-\alpha \log r .
$$

지프 법칙은 문서, 책, 댓글 등 언어학에서 성립하며, 그 외에도 도시의 인구 순위, 지진의 크기 빈도 등에서도 성립 한다는 사실이 잘 알려져 있다 [7-9].

\section{2. 상관관계}

두 데이터 사이에 얼마나 관계가 있는지 수치로 나타내 기 위해 상관관계를 사용한다 [10]. 양의 값은 두 데이터가 완전히 같은 경향을 보이는 경우이며 ( $X$ 가 증가할 때, $Y$ 도 같이 증가하는 경우), 음의 값은 완전히 반대의 경향을 보 이는 경우이다 ( $X$ 가 증가할 때, $Y$ 는 감소하는 경우). 0 은 두 데이터간의 관계가 전혀 없음을 의미한다. 두 집단 $X, Y$ 에 대하여 표본 상관계수 $r_{X, Y}$ 는 식 (3)과 같이 구한다.

$$
r_{X, Y}=\frac{\sum_{i=1}^{n}\left(X_{i}-\bar{X}\right)\left(Y_{i}-\bar{Y}\right)}{\sqrt{\sum_{i=1}^{n}\left(X_{i}-\bar{X}\right)^{2}} \sqrt{\sum_{i=1}^{n}\left(Y_{i}-\bar{Y}\right)^{2}}} .
$$

$\bar{X}, \bar{Y}$ 는 각각 $\mathrm{n}$ 개의 데이터 안에서의 평균값이다. 피 어슨 상관계수 (Pearson correlation coefficient)는 보통 $\left|r_{X, Y}\right| \geq 0.5$ 이면 높은 상관관계라 할 수 있다. 
Table 1. It is consisted with properties of Korean presidential speeches. The total speeches, the number of 6,870 , have 63,225 distinct tokens and its document length is $8,741,085$ words.

\begin{tabular}{lcccc}
\hline \hline & Speeches & Text volumes (KB) & Distinct tokens & Document length \\
\hline Syngman Rhee & 998 & 3,818 & 23,497 & 804,424 \\
Posun Yun & 3 & 8 & 635 & 1,524 \\
Chung-hee Park & 1,270 & 9,310 & 25,781 & $1,881,037$ \\
Kyu-hah Choi & 58 & 284 & 5,416 & 76,391 \\
Doo-hwan Chun & 602 & 4,164 & 16,515 & 824,632 \\
Tae-woo Roh & 601 & 5,722 & 21,966 & $1,140,380$ \\
Young-sam Kim & 728 & 4,146 & 16,272 & 804,139 \\
Dae-jung Kim & 822 & 5,569 & 19,228 & $1,096,115$ \\
Moo-hyun Roh & 780 & 4,734 & 18,983 & 938,352 \\
Myung-bak Lee & 817 & 4,716 & 22,665 & 926,560 \\
Geun-hye Park & 191 & 1,289 & 10,234 & 247,543 \\
\hline \multicolumn{1}{c}{ Total } & 6,870 & 43,854 & 63,225 & $8,741,085$ \\
\hline \hline
\end{tabular}

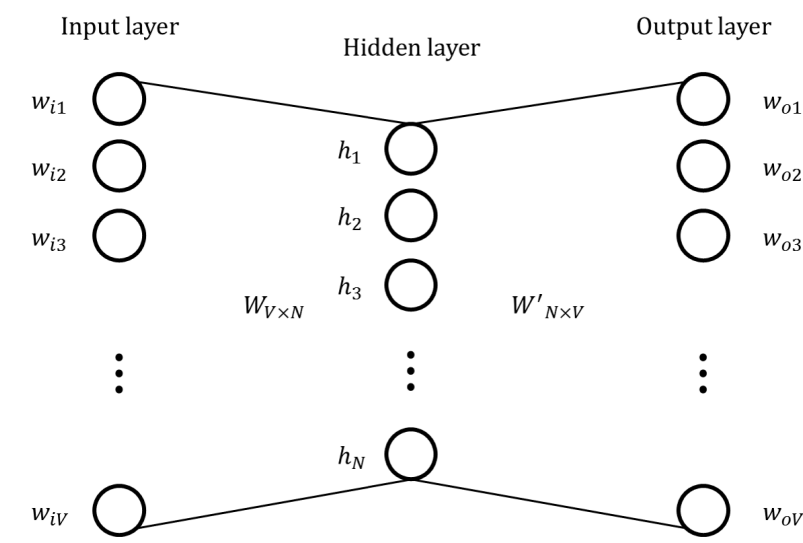

Fig. 1. Word2Vec algorithm is consist of three layers (input, output and hidden layer). Its operating method bases on the back propagation in recurrent neural network. $W_{V \times N}, W_{N \times V}^{\prime}$ between each layers is weight matricis which transform dimension of layer. Word2Vec print words to vector by the hidden layer.

\section{Word2Vec}

2013년 구글의 Mikolov팀이 개발한 Word2Vec은 단 어를 다차원 공간의 벡터로 변환시키는 알고리즘 (algorithm)이다 [4]. 이는 뉴럴 네트워크의 기반이 되는 역전 달 (back propagation)을 토대로 입력층 (input layer)과 출력층 (output layer) 사이에 단일층의 숨은 층 (hidden layer)을 둔 형태이다. Word2 Vec에서는 단일 숨은 층을 이 용하는데, 숨은 층의 크기를 정할 수 있으며 데이터를 통해 기계학습을 진행하고 최종적으로 그 숨은 층을 각각의 단 어벡터로 출력하는 방식이다 (Fig. 1).

물론, Word2 Vec가 전혀 새로운 방법론은 아니지만, 계층 소프트맥스 (hierarchical softmax), 음성 표본 추출 (nega- tive sampling)과 같은 최적화 알고리즘을 통해 다른 알고 리즘들에 비해 동작 시간을 단축시켰기 때문에 최근 주목 받고 있다 [5].

\section{IV. 결 과}

영어권에서 많이 이루어진 지프 법칙이 한국어의 경우 확인하는 것이 쉽지 않다는 사실이 잘 알려져 있다. 각 대 통령의 연설문은 일정 구간 [ 20 위, 2,000 위]에서 지프 법칙 (Zipf' law)이 성립하였다.

Fig. 2은 각각의 대통령에 대해 단어 순위별 빈도 확률 을 로그-로그 축척 (log-log scale)으로 나타내었다. 모든 연설문을 한 그래프에 그려내기 위해 빈도수는 각각 정규 화 (normalization)를 해주었다. 각각의 점들은 대통령별로 색을 다르게 그렸으며, 검은 실선은 그 기울기 $\alpha$ 가 1 인 거듭 제곱법칙을 따르는 경우를 나타내었다. 1 - 20위를 차지한 단어들은 감탄사, 조사, 어미, 그리고 문장 부호 등 문장의 구조를 이루는 성분들로서 그 빈도가 비슷하게 쓰이므로 1 보다 작은 값을 갖는다. 반대로, 빈도-순위 그래프에서 꼬 리 부분의 경우 (상위 2,000 위 이하)는 고유명사, 지정사와 같이 문서에서 많이 쓰이지 않아 표본이 적기 때문에 지프 기울기는 1 보다 큰 값을 갖는다.

년도별로 연설문 내 단어 빈도수 분포 변화의 상관관계 를 계산한 것을 Fig. 3 에서 나타냈으며, 각각의 임기기간 을 표시했다. 한 대통령의 임기기간 동안의 연설문 내에서 년도별 상관관계는 1 에 가까운 수치를 나타냈다. 하지만, 5-9대 박정희 대통령과 14 대 김영삼 대통령의 경우는 다른 양상을 보인다. 먼저, 박정희 대통령의 경우 총 5 번의 대 통령직을 수행해왔다. 연설문 내의 상관관계를 살펴보면, 그의 첫 임기기간과 그 후로 나뉘어짐을 볼 수 있다. 


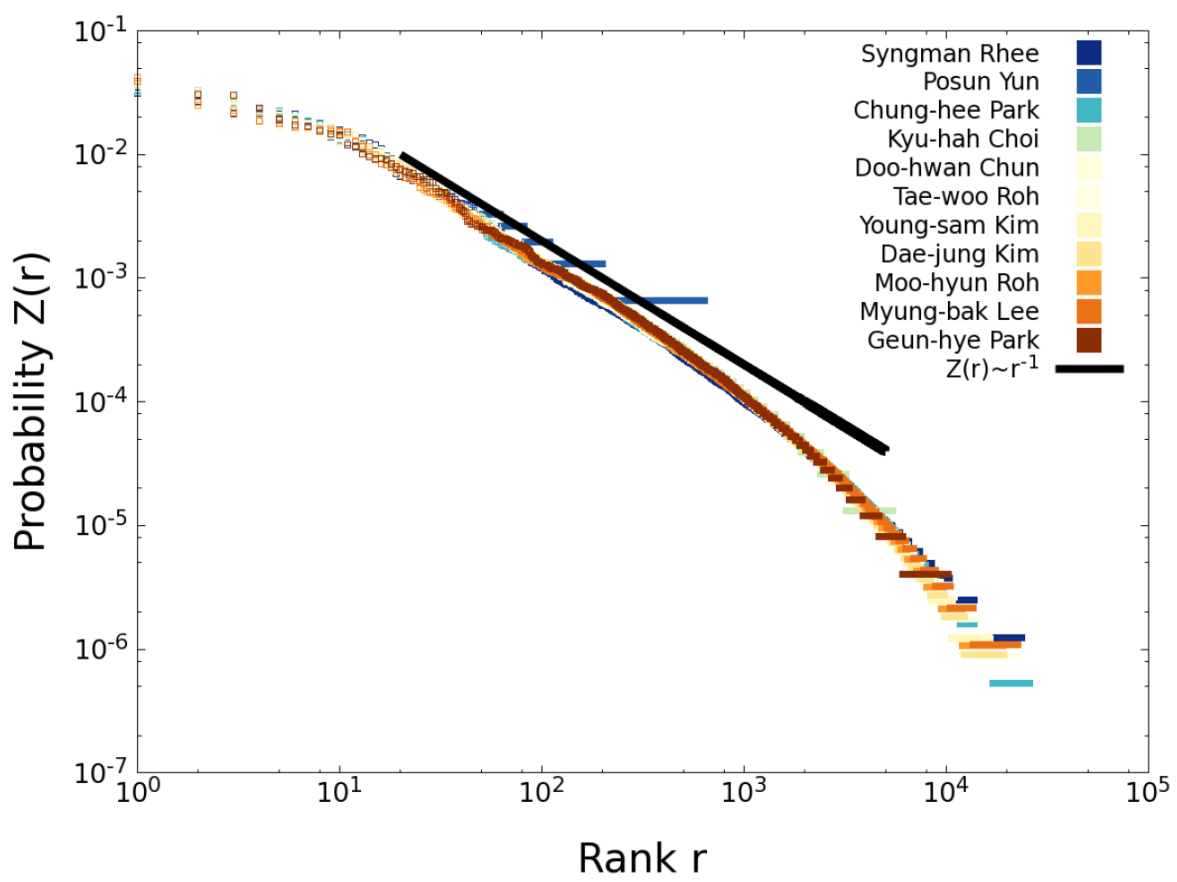

Fig. 2. (Color online) All speeches follow the Zipf's law in the partial range [20, 2,000]. Each axes is drawn by log-log scale. X-axis means rank of the word and y-axis means probability of the word. The slopes could be 1 .

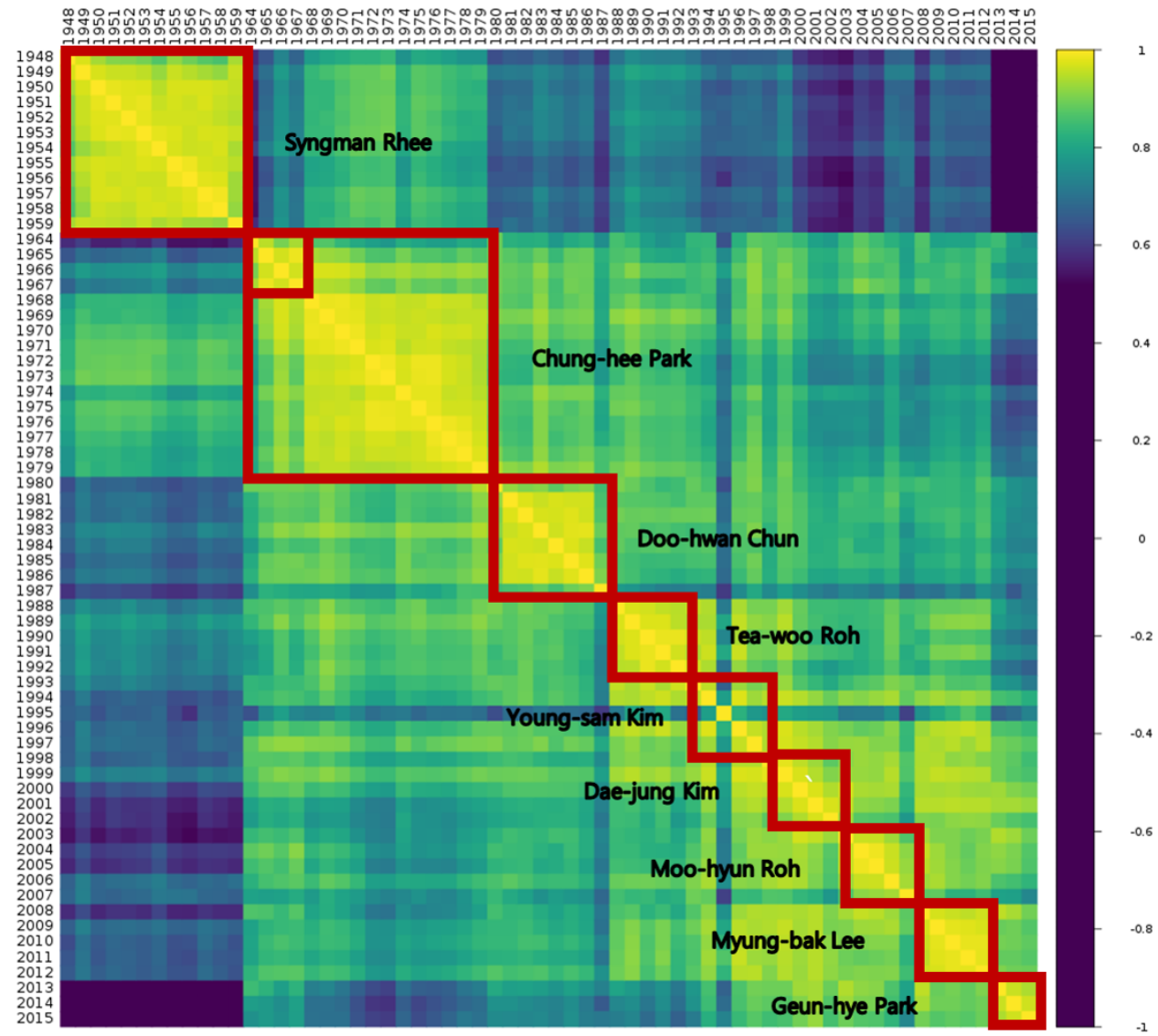

Fig. 3. (Color online) The correlation matrix between word-frequencies distribution of presidential speeches for each years. The correlation coefficient is 1 in yellow areas and -1 in dark blue areas. These coefficient during one presidential term are high. However, there are critical points in the era of Chung-hee Park and Young-sam Kim. 


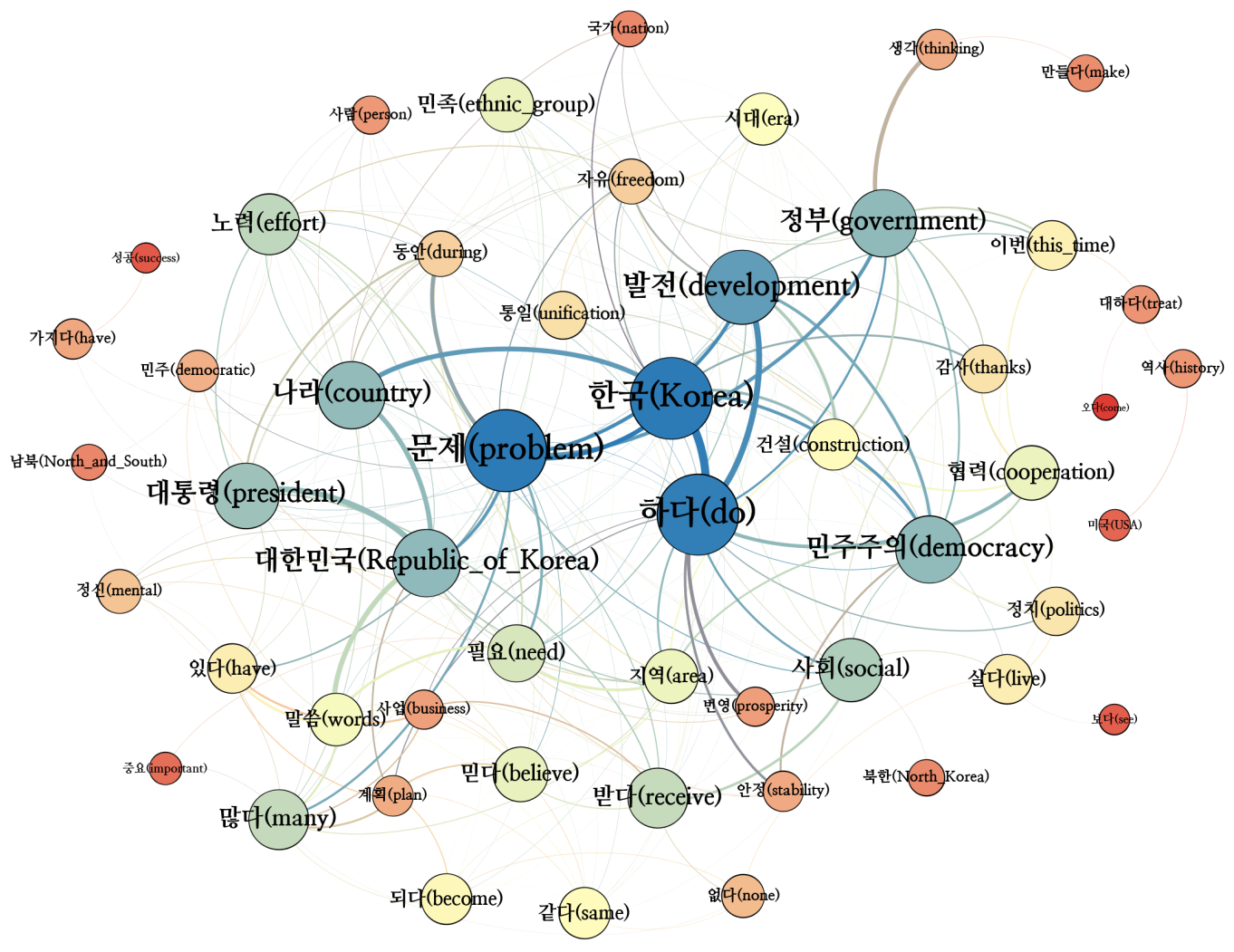

Fig. 4. (Color online) A network among words, which is the top 52 with the highest frequency in total speeches, in all of the presidential speeches is undirected, weighted network. Vertex show each words and the size is proportional to summation of its degree weight.

또한, 김영삼 대통령의 1995년 한 해는 다른 년도와 상 관관계가 확연히 다름을 알 수 있다. 상관관계를 분석함으 로써 특이점을 관측할 수 있다.

Word2Vec은 단어를 벡터 공간에 뿌려준다. Word2Vec 을 통해 얻어진 벡터값을 이용하여 각 단어간의 피어슨 상 관관계 (Pearson correlation coefficient)를 구하고, 이를 바탕으로 네트워크를 구성했다. Fig. 4은 시각화를 위해 전체 연설문에서 빈도수가 높은 상위 52 개의 단어들만을 구성하여 전체 연설문에 대해 나타내었다. 각 단어들을 정 점 (vertex)로 각각의 상관계수를 가중치로 하는 연결선 (weighted edge)을 부여했다. 한국의 대통령들은 전체적으 로 한국의 정부와 국민들의 문제를 생각하고 있다는 것을 볼 수 있다. 또한, 자유, 노력, 그리고 필요 등의 단어로 비 추어 볼 때, 민주주의를 향한 노력을 지속적으로 해왔음을 유추해 볼 수 있다.

\section{V. 토 의}

한국어 분석을 통해 한국어에서도 지프 법칙이 일정 구간 성립함을 보였으며, 크게 3 구간으로 나뉘어지며 각 구간에
서 나타나는 단어들의 특징을 살펴보았다. 년도별로 연설문 상관관계를 측정하였을 때, 각 시기별 (대통령별)끼리 높은 상관관계를 나타냈으며, 박정희 대통령과 김영삼 대통령의 연설문에서 특이점이 보였다. 실제 박정희 대통령은 초기 집권 시에는 내부 집권에 힘썼으며, 후기에는 대외적으로 활동하며 국력을 강화했음을 알 수 있다. 김영삼 대통령 연 설문의 경우, 김영삼 대통령이 1994년 마틴루터킹 센터가 수여하는 비폭력 평화상을 받았으며, 1995년 국제연합 50 주년 기념총회에서 연설을 하는 등 국제적인 이슈가 그의 연설문에도 큰 영향을 끼친 것으로 보인다. 네트워크를 통 해 연설문에서 어떤 단어들이 같이 쓰였는지와 연설문에서 쓰인 단어들의 연결망을 시각적으로 볼 수 있었다.

최근 대통령 연설에 많은 이슈를 불러 일으키고 있다. 한국어 텍스트 분석기법 또한 다양해지고 있다. 특히, 기계 학습을 통한 텍스트 분석은 정량적인 값으로 글을 분석할 수 있으며, 단어 연결망으로 나타내어 시각화할 수 있다. 나아가, 언론 기사나 소셜 커뮤니티 등의 여러 분야에서 언어를 분석할 때, 이러한 방법은 새로운 측면에서 텍스트 를 시각화하고 직관적 이해를 높일 수 있는 방법론이 될 것으로 생각된다. 


\section{감사의 글}

이 논문은 2017년 정부 (교육부)의 재원으로 한국연구 재단 (No. NRF-2014R1A1A2057396)과 포스코 청암재단 청암과학펠로십의 지원을 받아 수행된 기초연구 사업입니 다.

\section{REFERENCES}

[1] E. L. Park and S. Cho, in Proceedings of the 26th Annual Conference on Human and Cognitive Language Technology (Chuncheon, Korea, Oct 2014), p. 133.

[2] J. H. Park, E. L. Park and D. J. Jo, Korean Political Science Review 49, 27 (2015).
[3] Predidential archives, http://www.pa.go.kr (accessed Jan. 15, 2016).

[4] T. Mikolov, K. Chen, G. Corrado and J. Dean, arXiv :1301.3781 (2013).

[5] X. Rong, arXiv:1411.2738 (2014).

[6] P. Bak, How Nanture Works: The Science of Selforganized Criticality, Copernicus Series (Copernicus, New York, 1996).

[7] R. Lin, Q. D. Ma and C. Bian, arXiv:1412.4846 (2014).

[8] L. Lu, Z. K. Zhang and T. Zhou, arXiv:1202.2903 (2012).

[9] M. Gerlach and E. G. Altmann, New J, Phys. 16, 113010 (2014).

[10] B. Zhu and J. Yuan, J. Hydroinform. 17, 90 (2015). 